\title{
EDITORIAL
}

\section{A decade in drug discovery}

\author{
Nature Reviews Drug Discovery marks its tenth anniversary this month, providing \\ an opportunity to reflect on the evolution of the landscape of drug research and \\ development (RED).
}

"The current challenges facing the pharmaceutical
industry are unprecedented in its history. Perhaps fore-
most among these are the industry's lower revenue
growth, poor stock performance, the lowest number of
new chemical entity (NCE) approvals and the poor late-
stage R\&D pipelines prevalent throughout the industry."
As we look back on 10 years of publishing the journal,
it is apparent that this evaluation of the pharmaceutical
landscape could have resonated with readers familiar
with the field at any time during the past decade. In
fact, it comes from an article analysing the status of the
industry near the beginning of this period, which posed
the question of whether the pharmaceutical industry
could reduce the attrition rates that have led to soaring
R\&D costs and to a lack of new drugs to compensate for
the huge loss of revenues as older blockbusters fall over
the patent cliff.

The article is one of ten - drawn from each year of the past decade - that have been highly cited and downloaded from our website during this time, which we have brought together in a freely accessible collection (available at http://www.nature.com/nrd/collections/10/index. html). As with many other articles we have published, it reflects one of the journal's goals to not just provide authoritative reviews of advances in the science underpinning drug discovery and development but also present data and analysis on key strategic and business issues for the field overall, such as the evolution of therapeutic area marketplaces, partnering trends and key events in patent law (see page 12).

Another article in the collection (see page 17) provides data on changes in several industry indicators over the past decade, including market rankings of major companies and products, R\&D budgets and the number of projects at different phases of development. Keeping in mind the assessment of the challenges facing the industry quoted above, it is of concern that the article reports that the probability of products from a group of major companies making it to market from initiation of Phase I trials fell steadily from $10 \%$ in $2002-2004$ to $5 \%$ in 2006 2008 , the most recent period for which sufficient data were available. This indicates that at least up to this point, strategies implemented to address attrition rates were not sufficient. Moreover, this challenge is likely to have been made harder by one of the other major issues of the past decade - concerns about drug safety - and its impact on the stringency of the regulatory environment.

Nevertheless, as noted in our traditional yearly review on page 6 , there were at least some positive signs in 2011, with the number of new drugs approved last year by the US Food and Drug Administration - 30 at the time of writing - looking close to reaching its highest point in the past decade. Although a 1-year upturn is insufficient to draw conclusions about whether this might be the beginning of a more productive period, it is notable that some of the most exciting drugs among these are for oncology indications, including several for rare cancers. Rare diseases and oncology are two areas that have become a major industry focus in recent years, in part because it seems that developing products that represent substantial improvements on current treatments, based on genomics-derived targets, could at least be increasingly feasible in these fields, if not yet in others.

Furthermore, and illustrating another trend during the past decade - a growing willingness to work precompetitively on major challenges for drug R\&D - efforts are beginning on innovative collaborations to address one of the key factors thought to underlie high attrition rates: lack of validation of novel therapeutic targets ${ }^{2}$. Providing information and insights that could help to foster such multidisciplinary and multisector collaborations, as well as discussing their progress (such as in the most cited article in 2010, from an academia-industryregulator consortium on drug transporters ${ }^{3}$ ) has also been one of the journal's goals in the past 10 years. In closing, we would like to take this opportunity to thank all of the authors, referees, interviewees and readers who have contributed to the journal's success in this time. Looking to the future, we hope that after another decade of publishing the journal, it will be possible to reflect on comprehensive success in addressing the challenges highlighted at the opening of this article.

\footnotetext{
1. Kola, I. \& Landis, J. Can the pharmaceutical industry reduce attrition rates? Nature Rev. Drug Discov. 3, 711-716 (2004)

2. Paul, S. M. et al. How to improve R\&D productivity: the pharmaceutical industry's grand challenge. Nature Rev. Drug Discov. 9, 203-214 (2010).

3. Giacomini, K. M. et al. Membrane transporters in drug development. Nature Rev. Drug Discov. 9, 215-236 (2010).
} 